\title{
Intrinsic creep of a granular column subjected to temperature changes.
}

\author{
Baptiste Blanc and Jean-Christophe Géminard. \\ Université de Lyon, Laboratoire de Physique, Ecole Normale Supérieure de Lyon, CNRS, \\ UMR 5672, Laboratoire, ENS, Université, 46 Allée d'Italie, 69007 Lyon, France.
}

\begin{abstract}
Minute external pertubations, such as temperature variations, can lead to a creep of the fragile structures that are the granular piles. We report a study, resolved in space and time, of the dynamics associated with the slow compaction of a granular column submitted to thermal cycles. Avoiding the thermal dilations of the container, we observe that the material still creeps, even in absence of external mechanical perturbations. The latter intrinsic creep of the material exhibits several surprizing features: the compaction of the material is not homogeneous along the column height and sudden collapses involve the entire system even if one could expect arches to screen the mechanical interaction between regions far apart one from another. In addition, the analysis of the dependence of the flow-rate on the frequency of the temperature cycles suggests that the creep is mainly induced by the propagation of the temperature profile, slow temperature changes being less efficient than rapid ones in making the material flow.
\end{abstract}

PACS:

47.57.Gc : Granular Flow, 45.70.Cc : Static sandpiles; granular compaction, 62.20.Hg : Creep.

\section{INTRODUCTION}

Granular materials, a collection of solid grains, are athermal systems as the effects of the thermal agitation are negligible in comparison to those of gravity [1]. However, properties of granular materials, especially their mechanical stability, slowly evolve with time [2]. Several mechanisms have been proposed to explain the latter aging process, among them the slow creep of the material produced by uncontrolled temperature variations [3].

The ability of temperature changes to induce the creep of granular materials has recently attracted the attention of physicists. Uncontrolled temperature changes have been suspected of being responsible for large-scale static avalanches [4], identified to lead to large stress fluctuations at the base of a granular pile [5], or to lead to minute rearrangments of the texture as monitored electrically [6]. Temperature cycles have even been shown to induce a systematic increase in granular packing [7]. T. Divoux reviewed the available literature on the subject in $2010[8,9]$.

The effects of temperature changes are a question of practical importance as temperature changes could initiate a landslide [10], the collapse of a silo [11] and, even, be responsible for the segregation of solid particles at the surface of asteroids [12] due to a thermally activated brazil-nut effect [13]. Aiming at a better understanding of the underlying mechanisms, we studied the slow compaction of a granular column submitted to periodic temperature variations $[14,15]$. Here, we extend the former experimental results to local measurements and to a detailed analysis of the effects of both the dilation of the confining structure and the properties of the temperature cycles.

\section{EXPERIMENTAL SETUP AND PROTOCOLS}

The principle of the experiment is to impose periodic temperature changes to a column of granular material, while controlling, or avoiding, thermal dilations of the container in order to assess the intrinsic creep of the material.

A column of granular material is confined in a vertical tube of radius $\mathcal{R}$, whereas a thin heating wire is stretched along the system axis. The wire is heated periodically by injection of a periodic current at the frequency $\omega=$ $2 \pi f$. Provided that $\omega$ is large enough, the diffusion length $\mathcal{L} \sim \sqrt{D \omega} \ll \mathcal{R}(D$ stands for the diffusion coefficient of heat in the granular material), the container remains at constant temperature. The method thus avoids the dilation of the container, at play in Refs. [7, 15].

In the configuration of Ref. [14], the heating wire is free to elongate along the system axis. The typical shear strain associated with a temperature change of magnitude $\Delta T$ is of about $L K \Delta T /(k d)$, where $L$ stands for the length of the wire and $k d$ for the thickness of a shear band involving $k$ layers of grains having the diameter $d$. If the wire is sufficiently stretched (such that its equilibrium length at the higher imposed temperature is smaller than the imposed length), the wire does not elongate. The only remaining effect at the boundaries is the change in its radius, $r_{w}$, the displacement of the corresponding wall being of the order of $r_{w} K \Delta T$, where $K$ stands for the typical thermal dilation coefficient of the wire material. The typical associated compressive strain, $K \Delta T$, is thus drastically reduced, $L / d$ smaller than the former shear strain [14].

The experimental setup is inspired by the one presented in Ref. $[14,15]$ but presents many different specifications (Fig. 1). The core of the system consists of a vertical glass tube $(1.5 \mathrm{~m}$ in height, $15 \mathrm{~mm}$ in inner diameter) firmly fastened to a wall in the basement of 
the physics department, so as to insure that the system is not subjected to any significant mechanical vibration. The column is filled with a granular material consisting of spherical glass beads (Matrasur Corp., sodocilicate glass) up to a height $H$, initially of about $135 \mathrm{~cm}$. We use samples exhibiting a large range of grain diameter, $d=(425-600) \mu \mathrm{m}$, in order to avoid the cristallization of the system. In addition, an inlet (not shown in Fig. 1) located at the bottom of the column makes it possible to loosen the material thanks to an upward flow of dry air.

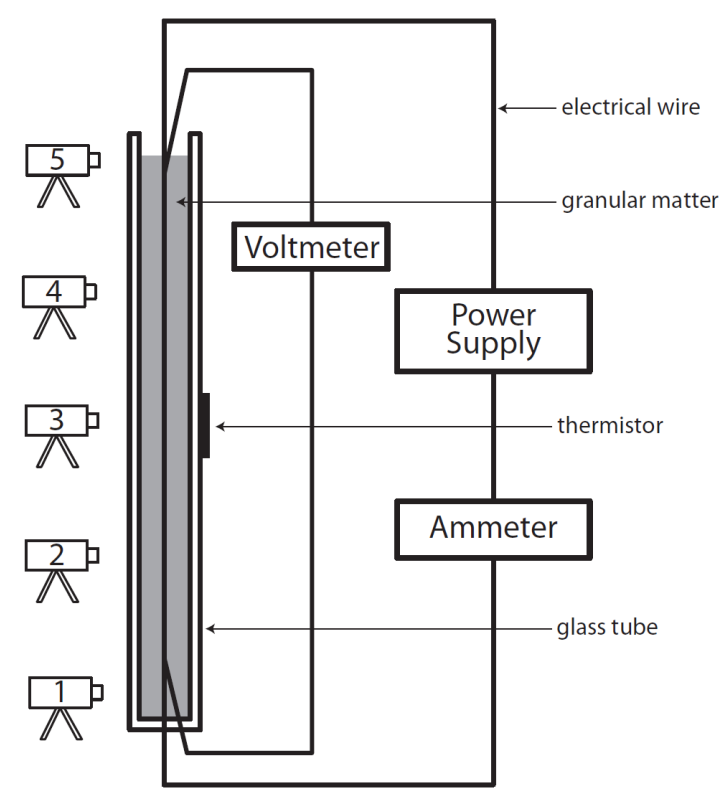

FIG. 1. Sketch of the experimental setup

In the former experimental configuration [15], the column was heated at the outer tube wall by means of a heating cable. The frequency $\omega$ of the temperature variations was chosen small enough to achieve a penetration length of about the tube radius and, thus, an almost homogeneous heating of the granular column. In the present configuration, the outer wall of the container is kept at a constant temperature. To do so, the whole system is placed in a wooden box $\left(50 \times 100 \times 200 \mathrm{~cm}^{3}\right.$ in depth, width and height) whose inner temperature is stabilized at $T_{0} \simeq 30 \mathrm{~K}$ to within $1 \mathrm{~K}$ thanks to a PIDcontroller (RKC, Rex S-100). Temperature changes are imposed to the granular material thanks to a thin conductor wire (Nickel, diameter $d_{w}=250 \mu \mathrm{m}$ ) placed along the axis of the tube. Injecting a well-controlled current $I(t)$ from a power amplifier (Kepco, 36-6M) driven by a data acquisition board (National Instruments, PXIe6124 ) and measuring the associated voltage $U(t)$ makes it possible to assess the instantaneous injected power $P(t)=U(t) I(t)$ and the temperature of the wire $T_{w}(t)$ at time $t$. Note that the voltage $U$ and the current $I$ are measured independently by means of two multime- ters (Agilent, 34410A), computer controlled (National Instruments, PCI-GPIB) and synchronized so as to insure that both quantities are obtained at the same time $t$. The temperature of the wire is deduced from the resistance, $R=U / I$, which depends on $T_{w}(t)$ according to $R=R_{0}+\eta\left(T_{w}-T_{0}\right)$ with $\eta=(2.01 \pm 0.01) 10^{-3} \Omega / \mathrm{K}$ and $R_{0}=(0.5274 \pm 0.0001) \Omega$. The latter resistancetemperature relation has been obtained by changing the temperature of the entire setup and by measuring, in the steady state, the corresponding resistance of the wire in place.

The granular material is imaged from the side thanks to 5 webcams (Logitech, Quickcam pro 9000) located at different heights along the column, $h_{1}=5, h_{2}=35, h_{3}=$ $64, h_{4}=92, h_{5}=134 \mathrm{~cm}$, the reference being at the bottom of the granular material. A light source, consisting of a powerful green LED (Kingbright, L-7104VGC-H), is associated with each of the cameras. The uppermost camera images the free surface of the granular column and makes it possible to assess the temporal variation of the total column height, $\Delta h_{5}(t)$, to within $25 \mu \mathrm{m}$, estimated from the scattering of the data. The four remaining cameras are used to assess the displacement of the grains at the container wall. To do so, images from all the cameras are taken at each temperature cycle, recorded during the whole experimental time and subsequently analyzed. The average displacement of the grains along the vertical at a given height is obtained from the correlation between the corresponding images ; depending on the local flow velocity, correlation between sucessive images or images separated by a large number of temperature cycles are considered. We determined that the best option was to consider images such that the average displacement of the grains between them is of the order of $20 \%$ of the grain diameter. The time difference between the images depends on the creep rate. The method insures a good temporal resolution and avoids the drift of the measurements at long time. On the one hand, if the system collapses between to successive images, we obtain the displacement with the best temporal resolution (The delay between two images, $1 / f$ ). On the other hand, for small creep rates that are associated with tiny, almost zero, displacements between successive images, we measure accurately the total displacement after a sufficiently long time. The final resolution of the measurement is of about $10 \mu \mathrm{m}$.

The experimental protocol, except when specified, is as follows. A given amount of grains, usually $370 \mathrm{~g}$, is poured in the container and air is injected at the base in order to obtain a loose packing. When the gas injection is stopped, the packing is loose and the initial position of the free surface of the granular material is high in the image from the uppermost camera. Then, the wooden chamber is closed and the system is submitted to periodic temperature variations by imposing a periodic current $I(t)=I_{0}+\delta I \cos \omega t$. Note that, the injected power, $P(t)=R I^{2}(t)$, exhibits a mainly harmonic modulation at the frequency $\omega$ only if $\delta I \ll I_{0}$ and if the changes in 
the resistance of the wire are small. In this latter conditions, $P(t) \simeq P_{0}+\delta P \cos \omega t$ with $\delta P \simeq 2 R_{0} I_{0} \delta I$, the higher-order terms being negligible. We shall not work strictly in this limit, using large amplitude of the current variations in order to get significant effects, but we mention here that, because of the ratio $\delta I / I_{0}$ of the amplitudes of the second and first harmonics in the injected power, the fundamental is the dominant thermal perturbation imposed to the system. After a transient of about 10 minutes, the thermal system reaches a steady state. The amplitude $\delta T$ of the temperature cycles is obtained by measuring $I$ and $U$, thus $R(t)$.

Hours after the cycling started, the delay depending on the amplitude $\delta T$ and on the frequency $f \equiv \omega /(2 \pi)$, the free surface of the granular material reaches a given level in the field of the uppermost camera. The corresponding filling fraction is then of about $60.4 \%$ (to be compared to the maximum filling fraction obtained by manual tapping $62.7 \%$ ). We estimated the diffusion length $\mathcal{L}$ from the value of the diffusion coefficient $D=210^{-7} \mathrm{~m}^{2} / \mathrm{s}$ [16], and checked by the use of a temperature sensor glued to the outside wall, that the temperature of the tube wall remains constant in our experimental range of frequency (e.g. for frequency much larger that $1 \mathrm{mHz}$ ).

We report on the subsequent evolution of the system.

\section{RESULTS}

\section{A. Irregular settling and spatial coupling}

Previous experimental results, obtained by heating periodically the container wall and assessing the total height of the granular column only, showed that the material can settle in a series of successive collapses separated by randomly-distributed times when the amplitude of the temperature cycles is below a given threshold [15]. The same behavior had already been observed in an even older experiment $[8,14]$, in which the granular material was heated by means of a heating wire along the axis, alike in the present experiment. One can wonder if the apparent collapses seen at the free surface were not the result of the emergence of density bubbles [17], initially trapped in the bulk of the material during the preparation of the initial condition, rising under the effect of the temperature variations. In this case, one would observe waves travelling along the vertical.

In order to elucidate the nature of the collapses of the granular surface, we perform a series of experiments in experimental conditions similar to those in Ref. [14]. The heating wire is simply clamped, at $T_{0}$, at the two ends of the vertical column. We point out that, with this experimental condition, the equilibrium length of the heating wire, which is at a temperature larger than $T_{0}$ during the whole temperature cycle, is always larger than the distance between the fasteners because of the thermal dilation. The wire is thus not stretched and likely to meander between the grains.

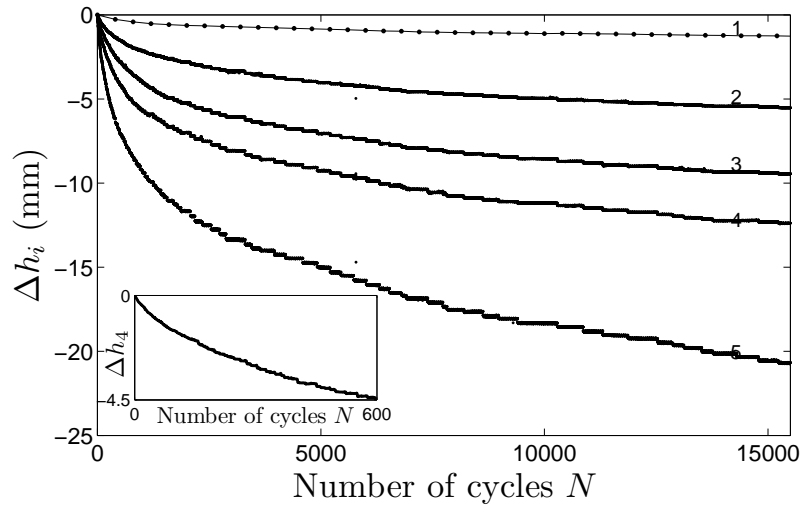

FIG. 2. Height variations $\Delta h_{i}$ vs. number of cycles $N$ - We observe that the granular colummn settles in a series of sudden collapses separated by quiescent periods. Sudden events are simultaneous in the image from all the cameras. Note that the total duration of the experiment is of about 7 days and that a compaction by $1 \mathrm{~cm}$ corresponds to a variation of $0.4 \%$ of the filling fraction. Inset : At short times, we observe that the material flows continuously in the same experimental conditions before entering the intermittent regime $(f=0.02 \mathrm{~Hz}$ and $\delta T=10 \mathrm{~K})$.

We report the vertical displacements $\Delta h_{\mathrm{i}}$ as a function of the number, $N$, of temperature cycles experienced by the system (Fig. 2), which is equivalent to report the data as a function of time. First, we observe that, as time passes by, the granular material settles all along its height and that the displacements measured at the side walls are larger at a higher vertical position. More precisely, the latter displacements are not proportional to the altitude, which proves that the compaction of the granular material is not homogeneous along the vertical, even far from the lower and upper boundaries. Note that the camera 5, at the top, images the free surface and, thus, does not inform us about the displacement of the grains at the side wall, but about the displacement of the free surface, in other words the average vertical velocity of the grains across the whole section of the container. We checked that motion of the free surface is about $15 \%$ larger than the motion of the grains at the wall, which means that the compaction of the column is mainly due to losts of stability and slidings at the walls, resulting in an almost solid-body downwards motion. Second, considering the details of the settling dynamics, we observe that, after a transient during which we observe a continuous flow (Fig. 2, inset), the granular material settles in a series of sudden collapses separated by quiescent periods. Moreover, within the temporal resolution of the observations (one image per cycle), the displacements measured at all heights exhibit simultaneous jumps.

One could wonder if the coupling along the vertical, and even the compaction, could not be induced by sudden rearrangments of the heating wire in the bulk of the granular material. In the next section, we thus discuss in 
details the effects of distinct fastening conditions.

\section{B. Extrinsic mechanical perturbations}

In the two aforementioned experiments [14, 15], the heating wire or the external wall are dilating. In order to evaluate the effects of these extrinsic mechanical perturbations we perform experiments with three different mechanical configurations:

1. - The heating wire is initially clamped at its two ends at room temperature $T_{0}$. When the temperature cycles are imposed, the average temperature of the wire is larger than $T_{0}$, the natural length of the wire, because of the thermal dilation, is larger than the distance between the fasteners at the two ends. The wire is likely to slither between the grains.

2. - The heating wire is clamped at the top and maintained at a constant tension thanks to a weight, a few tenth of grams, hanging at the bottom. When the temperature cycles are imposed, the wire remains stretched along the axis and is likely to induce a shear deformation, increasing linearly with the distance to the free surface.

3. - The heating wire is clamped at the bottom and maintained at a constant tension thanks to a spring at the top. When the temperature cycles are imposed, the wire remains stretched along the axis and is likely to induce a shear deformation, increasing linearly with the distance to the bottom.

For the three mechanical configurations, the preparation of the system is identical: Starting from a loose system, consisting of the same amount of grains, high frequency cycles $(f=1 \mathrm{~Hz})$ are imposed until a given total height $h_{5}$ of the granular column is achieved. We report the subsequent variation of the total height of the granular column, $\Delta h_{5}$, as a function of time $t$ (Fig. 3 ).

First, let us mention that, contrary to what was observed when the container was heated and, thus, the wall dilating, the free surface does not exhibit any significant vertical oscillation at the frequency of the temperature variations : The filling fraction being always far from maximum, the heated grains rearrange and find the necessary free space locally ; There is thus no cumulative effect leading to any significant vertical displacement of the free surface.

Second, we observe that the granular column settles faster for the configuration 2 , in which the dilation of the wire induces a shear of the material at the bottom, than for the configuration 3, in which the shear is mainly induced at the top. The configuration 1, which corresponds to a wire likely to slither between the grains, is an intermediate case. One could wonder why the efficiency of the wire is so different when clamped at the top or at the bottom. Here, we can attempt to give a potential explanation of the observed difference. Note first that, in both cases, due to the stretching imposed by either the mass or the spring, the wire remains straight and only elongates along the vertical axis. For the same amplitude of the temperature cycles, the amplitudes of the associated displacements at the top, in one case, and at the bottom, in the other case, are the same. However, the situations are clearly not symmetric. For instance, in the configuration 2 , the wire, clamped at the top, reaches the maximum length when the temperature is maximum and thus starts moving upwards at the largest temperature. To the contrary, in the configuration 3 , the wire, clamped at the bottom, reaches the minimum length when the temperature is minimum and thus starts moving upwards at the smallest temperature. A larger temperature being associated with a larger radial stress confining the heating wire, one can understand why the two situations, even if they are associated with the same shear, are not symmetric.

The shear at the bottom (situation 2) is very efficient in making the system settle. However, the experiment is very sensitive to the preparation of the granular packing, which leads to a lack of reproductibility from one run to another and which thus makes it difficult to precisely assess the relative efficiency of each of the conditions. From these observations, we shall only remember that they clearly reveal that the mechanical disturbances originating from the dilation of the wire play an important role in the compaction process.

One could now wonder if the granular material settles in absence of external mechanical disturbance, which is the subject of the next section III C.

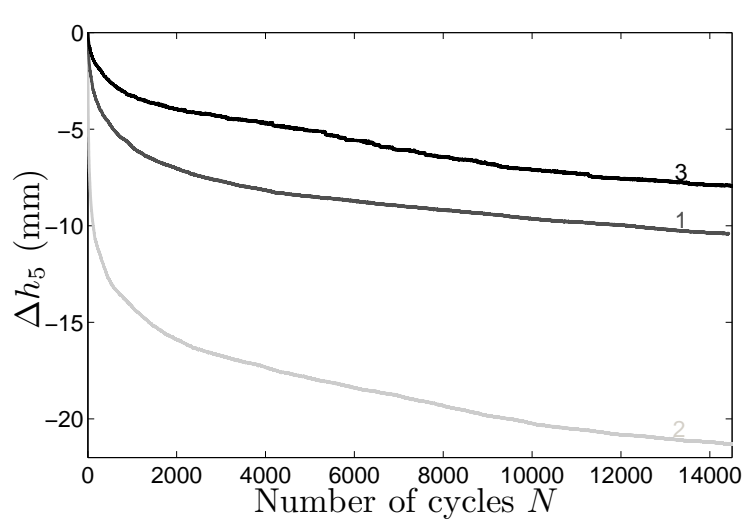

FIG. 3. Height variation $\Delta h_{5}$ vs. time $t-$ For the same preparation protocol of the material and thermal cycles, the dynamics of the granular column is sensitive to the fastening condition of the heating wire: the compaction is faster when the heating wire induces shear at the bottom (config. 2) than at the top (config. 3). A heating wire likely to slither between the grains (config. 1) constitutes an intermediate case $(f=$ $0.02 \mathrm{~Hz}$ and $\delta T=10 \mathrm{~K})$. 


\section{Intrinsic creep of the granular material}

In order to avoid most of the mechanical perturbations induced by the heating wire, the latter is now initially stretched and clamped at its two ends at a large preparation temperature. In this case, when the temperature cycles are imposed, the natural length remains smaller than the distance between the fasteners. The wire, which thus remains stretched, does not impose any vertical displacement to the grains. In this case, the perturbations induced by the walls are drastically reduced. Indeed, formerly, the dilation of the heating wire was producing a displacement along the symmetry axis of about $10 \mu \mathrm{m} / \mathrm{K}$, the dilation over $1 \mathrm{~m}$, at one end to be compared to the only remaining contribution due to the change in the wire radius which is of the order of $1 \mathrm{~nm} / \mathrm{K}$. The associated strains are respectively of the order of $10^{-5} / \mathrm{K}$ and $510^{-3} / \mathrm{K}$ (Sec. II). In addition, provided that the frequency is large enough for the penetration length, $\mathcal{L}$, to be smaller than the radius of the tube, the outer wall remains at constant temperature and does not induce any mechanical perturbation.

We observe that the granular material, even if the external mechanical perturbations have been drastically reduced, settles when submitted to temperature changes (Fig. 4). The effect is significantly smaller and the high frequency cycles $(f=1 \mathrm{~Hz})$ imposed during the preparation of the system were not sufficient to achieve, in an acceptable time, the chosen initial granular height $h_{5}$. Thus, in order to report results in this configuration, the system is prepared by making the granular packing loose thanks to the ascending gas flow from the inlet. The material is then slightly compacted by the application of mechanical taps from an electromagnetic shaker to achieve the chosen column height. The temperature cycles are then directly applied without any further preparation of the system. We report the displacement of the grains at the tube wall at various heights (Fig. 4).

First, we observe that the displacements are not proportional to the height above the bottommost part of the column, which indicates that the compaction is not homogeneous. Second, it is particularly interesting to notice that the temporal evolution of the displacements $\Delta h_{\mathrm{i}}$ at various heights are correlated. Indeed, in the right panels in Fig. 4 one clearly observes that the sudden collapses are simultaneous in both panels, which reveals that the two regions are mechanically coupled. This result is rather surprizing as the distance between the regions imaged by the cameras is about 15 times the container diameter. One would expect the redirection of the forces toward the walls [21] to be at play and the regions to be rather independent. The result remains unexplained.

In order to assess the efficiency of the thermal cycles, we naturally reproduced the experiments at various amplitudes and frequencies of the thermal cycles. However, we immediately noticed that, in spite of a well-defined preparation protocol, the flow curves were largely scattered for the same experimental parameters (Fig. 5). Es-

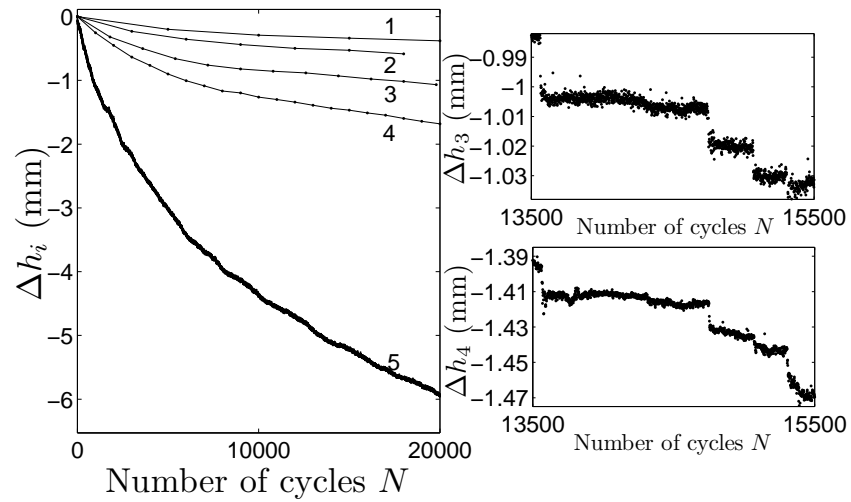

FIG. 4. Height variations $\Delta h_{i}$ vs. number of cycles $N$ - Left panel - We observe that the displacements are far from being proportional to the distance to the bottom, which indicates that the compaction is not homogeneous along the column. The sudden collapses are barely visible when the data are presented to assess the behaviour at long time. Right panels - The sudden collapses are simultaneous in the images from the cameras 3 and $4(f=0.02 \mathrm{~Hz}$ and $\delta T=10 \mathrm{~K})$.

tablishing precisely the law relating the flow rate to the characteristics of the temperature cycles is barely feasible. Indeed, due to the duration of the experimental runs (one and a half day for the shortest at $0.1 \mathrm{~Hz}$ up to more than a week for the longest), one cannot reproduce the experiments a sufficiently large number of times to get significant averages.

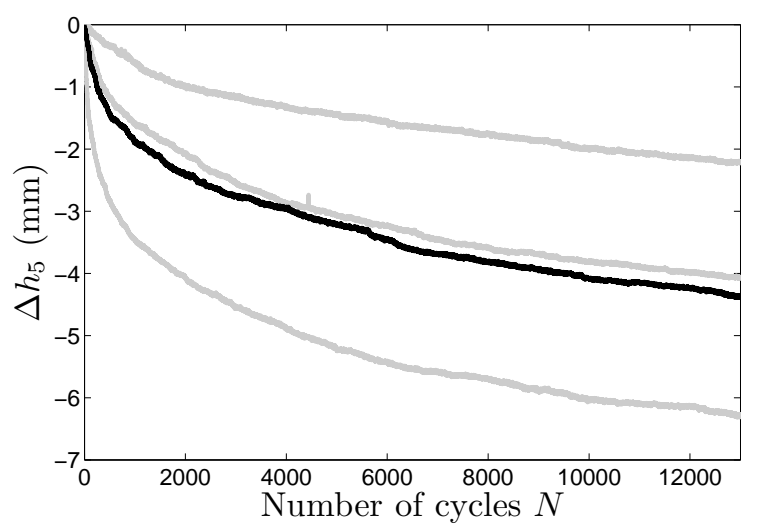

FIG. 5. Height variation $\Delta h_{5}$ vs. time $t$ - Starting from different preparations with the same protocol, we observe a large scattering of the compaction curves $(\delta T=7.6 \mathrm{~K}$, grey $0.1 \mathrm{~Hz}$ and black $0.05 \mathrm{~Hz}$ ).

Thus, to study the efficiency of different amplitudes $\delta T$, at a given frequency $f$, we start from a unique initial state and change the amplitude during the experimental run (Fig. 6). We observe that, obviously, a larger amplitude leads to a faster creep. We also notice that the creep rate seems to vanish at finite amplitude, in agreement 
with previous experimental results [15] and theoretical expectations from a toy frictional system [23].

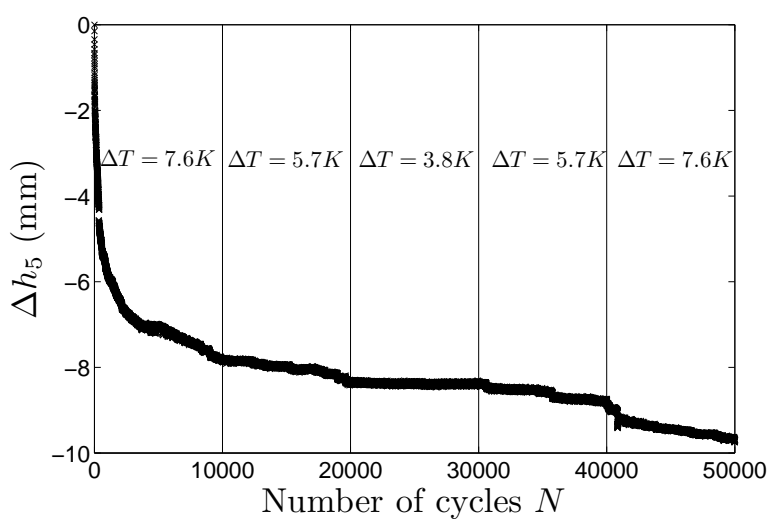

FIG. 6. Height variation $\Delta h_{5}$ vs. time $t-$ We observe that the compaction is faster for larger amplitude, as is clearly demonstrated by the change in the curvature of the curves when the amplitude is increased. We also observe that the creep velocity vanishes at a finite amplitude, of about $3 \mathrm{~K}$ $(f=0.1 \mathrm{~Hz})$.

The dependence of the compaction dynamics on the cycling frequency is obtained following a similar protocol. However, in order to distinguish the effects of the frequency from those of the amplitude, a special attention is paid to maintain the cycles amplitude constant while the frequency is changed. In addition, we consider an amplitude of the thermal cycles $(\delta T=7.6 \mathrm{~K})$ such that the temperature variations are reasonably sinusoidal and the frequency well-defined. During a single experimental run, we change the frequency of the temperature cycles, maintaining thus the amplitude $\delta T$ constant, and observe the associated dynamics (Fig. 7). Note that, at constant amplitude, the compaction rate (the height variation $\Delta h_{5}$ per applied temperature cycle $N$ ) decreases monotically when $N$ increases. Changing the frequency, we observe that an increase of the driving frequency leads to an increase of the creep rate. The latter experimental observations suggest that the thermal cycles are more efficient at larger frequency.

\section{DISCUSSION AND CONCLUSIONS}

The present experimental study provides several clues for the understanding of the creep of the granular matter submitted to temperature changes but leaves several open questions.

The main progress is to have observed the creep of the granular material in absence of significant external mechanical perturbation by avoiding, by contrast with the previous studies, the dilation of the container and by reducing the strain due to the heating wire at the center. The study of various mechanical configurations reveals

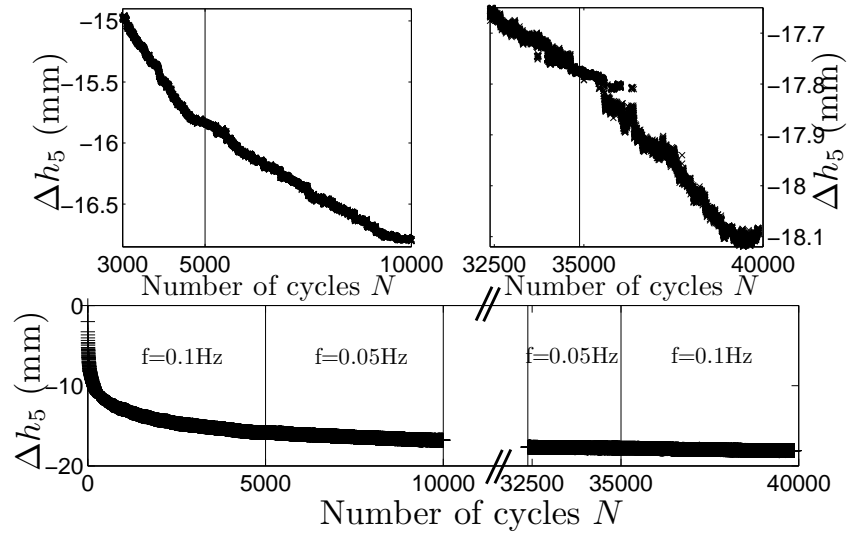

FIG. 7. Height variation $\Delta h_{5}$ vs. time $t-$ We observe that (a) the creep rate decreases when the frequency is decreased and, conversely, (b) increases when the frequency is increased. The lower panel displays the whole behaviour of $\Delta h_{5}$ vs. time $t$ from which the data of the upper panels are extracted $(\delta T=7.6 \mathrm{~K})$.

the important role played by the dilations of the external walls or of the heating wire in the former experiments [8].

Using several cameras to assess the displacements of the grains at the external wall in various regions along the column height, we observe that, in absence of external mechanical perturbation, the compaction of the material is not homogeneous. The observation is compatible with numerical results to be published elsewhere [24]. The experiment also shows that the large and sudden collapses of the free surface are not due to the emergence, at the top of the column, of density bubbles rising up in the bulk of the granular material. Indeed, during the preparation of the initial state, air is blown through the granular material in order to obtain a loose packing. Density bubbles (large regions of smaller density) are observed during this stage and one could expect regions of smaller density to have remained trapped in the granular material when the air injection was stopped. Subsequently, when the granular creep is induced by the temperature changes, one could expect the latter density bubbles to rise up slowly (Contrary to what is observed in dynamical experiments in which the granular material is fluidized [17-19], we expect the dynamics of the bubbles to be very slow in a frozen material. We do not refer here to the rapid propagation of quake waves [20] originating at the bottom). In such a picture, bubbles would rise slowly and emerge sequentially at the free surface, leading to the intermittent dynamics observed at the top of the column $[8,15]$. Our observations are clearly in contradiction with the latter picture because the large collapses are simultaneously observed in the images from all the cameras. They are in agreement with previous observations of quake waves all along the column height [20]. Note that this result is surprizing as one would expect the redirection of the forces toward the lateral walls $[21,22]$ to screen the long- 
range correlations between different heights. Indeed, in our system, the collapses can originate anywhere along the column height. We can barely conceive quake waves propagating easily from top to bottom but we do not observe more collapses at the top than at the bottom.

Interestingly, the experimental results show that the creep-rate, i.e. the height variation per cycle, increases with both the amplitude and the frequency. Whereas the increase with the amplitude is expected, the increase with the frequency is not obvious and deserves to be thoroughly commented here. Note first that maintaining the amplitude of the thermal cycles constant insures that the mechanical effects due to the heating wire are the same at any frequency (The temperature variation and, thus, the thermal dilation of the wire are the same whatever the frequency). Thus, the dependence of the creep rate on the frequency is not due to any effect associated with the heating wire and informs us about the mechanisms potentially involved in the process.

Faster temperature changes, associated with the propagation of sharper temperature profiles inside the material, are more efficient. The corresponding temperature gradients are associated with dilation gradients, thus to a shear of the material. At this point, it is interesting to consider some orders of magnitude. A temperature difference $\delta T$ leads to a typical strain of about $K \delta T$, in a region of typical size $\mathcal{L}$ where the penetration length $\mathcal{L}$ is of the order of $\sqrt{D / \omega}\left(K \sim 1.510^{-5} / \mathrm{K}\right.$ is the dilation coefficient and $D \sim 210^{-7} \mathrm{~m}^{2} / \mathrm{s}$ the thermal diffusion coefficient). Considering the granular material at the grain scale, we estimate the relative displacement, $u \sim[K \delta T / \mathcal{L}] d^{2}$, of two grains at the contact point under the action of the relative dilation of the grains (temperature gradient $\delta T / \mathcal{L}$ ) and compare the latter to the typical size of an asperity at the grain surface, $\delta$, which is of the order of a few nanometers [25]. At $f=0.01 \mathrm{~Hz}$, we get $u \sim 2 \mathrm{~nm}$ for $\delta T=1 \mathrm{~K}$. In the latter picture, the temperature gradients produce, at the contact points, relative displacements of the grain surfaces over distances that compare to the size of the asperities and, thus, induce rearrangments at the contact level and a creep of the material. Considering that the distance $u \propto \sqrt{\omega} \delta T$, we recover the experimental feature that the creep is enhanced by larger amplitudes $\delta T$ and frequencies $\omega$. Moreover, one can predict a finite threshold amplitude, at a given frequency, below which the material stops creeping. From the estimates above, the latter threshold amplitude is found to be of the order of a few degrees, as ob- served experimentally. Note, however, that temperature changes of the same amplitude are associated with dilations of the grains able to produce large static avalanches in models that do not invoke the roughness of the grain surface [4].

To go farther, we comment that the picture above also can account for a second onset in the amplitude separating the continuous- and the intermittent-flow regimes. Indeed, if the amplitude is such that the displacement $u$ is much larger that the typical scale of roughness, one can expect each cycle to destabilize the granular packing and, thus, the material to flow continuously (a displacement is associated to each cycle). Experimentally, we never reached such regime over a long time. However, we can guess that, in the initial regime, the system presents a large number of weak contacts (stabilized by small asperities), which would explain the initial continuous-flow regime (Fig. 2, inset). As time passes by, weaker contacts are statistically eliminated and single cycles become unable to induce a significant internal avalanche [26]. We observed (not shown) that the initial transient leads to a typical size of the jumps after a given number of cycles (which depends on the cycles characteristics) and not for a given average density. This observation indicates that the system is very sensitive to the texture of the contact network which partly results from the temperature cycles themselves.

Finally, we note that, in accordance, individual experimental runs are barely reproducible even when an identical preparation protocol is used to prepare the initial state. This experimental feature clearly indicates that the average density is not sufficient to define the state of the granular packing. In the present experimental configuration, we are missing at least a second experimental parameter, likely the internal stress or pressure, to precisely define the state of the system. Indeed, a granular column can exhibit apparently equal states (same density) having different response to mechanical perturbations [27]. This conclusion leads to propose another experimental configuration in which the stress is precisely controlled, which we now achieve by studying the creep of a granular layer laying on an incline.

\section{ACKNOWLEDGMENTS}

The authors acknowledge the financial support from the Agence Nationale de la Recherche, France (Contract \# ANR-09-BLAN-0389-01).
[1] E. Weeks, Soft jammed materials, in Statistical physics of complex fluids, Eds S. Maruyama, M. Tokuyama, 2.12.87, Tohoku University Press, Sendai (2007).

[2] L. Bocquet, E. Charlaix, S. Ciliberto, and J. Crassous, Nature London 396, 735 (1998).

[3] J.-C. Géminard and E. Bertin, Phys. Rev. E 82, 056108
(2010).

[4] P. Claudin and J.-P. Bouchaud, Phys. Rev. Lett. 78, 231 (1997).

[5] L. Vanel and E. Clément, Eur. Phys. J. B 11, 525 (1999).

[6] D. Bonamy, L. Laurent, P. Claudin, J.-P. Bouchaud and F. Daviaud, Europhys. Lett. 51, 614 (2000). 
[7] K. Chen et al, Nature 442, 257 (2006).

[8] T. Divoux, Papers in Physics 2, 020006 (2010).

[9] A. Coniglio and M. Pica Ciamarra, Papers in Physics 2, 020007 (2010).

[10] B. Voight and D. Elsworth, Geotechnique 47, 1-31 (1997).

[11] J. W. Carson and T. Holmes, T.A.S.K. quarterly 7, 499512 (2003).

[12] P. Sanchez, M. R. Swift and D.J. Scheeres, Granular Mechanics in the Asteroid Regime, AAS/Division for Planetary Sciences Meeting Abstracts, 41, 27.13 (2009).

[13] K. Chen, A. Harris, J. Draskovic and P. Schiffer, Gran. Matt. 11, 237-242 (2009).

[14] J.-C. Géminard, Habilitation à Diriger des Recherches, Université Joseph Fourier, Grenoble I, p. 32 (2003). http://tel.archives-ouvertes.fr/tel-00294761/fr/

[15] T. Divoux, H. Gayvallet and J.-C. Géminard, Phys. Rev. Lett. 101, 148303 (2008).

[16] J.-C. Géminard, and H. Gayvallet, Phys. Rev. E 64, 041301 (2001).
[17] Y. Bertho, F. Giorgiutti-Dauphine and J.-P. Hulin, Phys. Fluids 15, 3358-3369 (2003).

[18] L. Bonneau, T. Catelin-Jullien and B. Andreotti, Phys. Rev. E 82, 011309 (2010).

[19] T. Börzsönyi and Z. Kowàcs, Phys. Rev. E 83, 032301 (2011).

[20] C. Wensrich, Powder Tech. 127, 87-94 (2002).

[21] H. A. Janssen, Zeitschr. d. Vereines deutscher Ingenieure 39,1045 (1895).

[22] M. Sperl, Gran. Matt. 8, 59-65 (2006)

[23] B. Blanc, L.A. Pugnaloni and J.-C. Géminard, Phys. Rev. E 84, 061303 (2011).

[24] B. Percier and N. Taberlet, private communication (2012).

[25] H. Alarcón, J.-C. Géminard and F. Melo, Phys. Rev. E 86, 061303 (2012).

[26] A Kabla and G. Debregeas, Phys. Rev. Lett. 92, 035501 (2004).

[27] E.R. Nowak et al, Powder Tech. 94, 79-83 (1997). 